Relations industrielles

Industrial Relations

\title{
Nationalism and the Multinational Enterprise, by R.H. Hahlo, G. Smith et R. Wright, New York, Oceana Publications Inc., 1973, 373 pp.
}

\section{Gérard Garnier}

Volume 28, numéro 4, 1973

URI : https://id.erudit.org/iderudit/028463ar

DOI : https://doi.org/10.7202/028463ar

Aller au sommaire du numéro

Éditeur(s)

Département des relations industrielles de l'Université Laval

ISSN

0034-379X (imprimé)

1703-8138 (numérique)

Découvrir la revue

Citer ce compte rendu

Garnier, G. (1973). Compte rendu de [Nationalism and the Multinational Enterprise, by R.H. Hahlo, G. Smith et R. Wright, New York, Oceana Publications Inc., 1973, 373 pp.] Relations industrielles / Industrial Relations, 28(4), 890-892. https://doi.org/10.7202/028463ar

Tous droits réservés (C Département des relations industrielles de l'Université Laval, 1973
Ce document est protégé par la loi sur le droit d'auteur. L'utilisation des services d'Érudit (y compris la reproduction) est assujettie à sa politique d'utilisation que vous pouvez consulter en ligne.

https://apropos.erudit.org/fr/usagers/politique-dutilisation/ 
A ce titre, Industrial Relations Systems de Dunlop demeure un classique et c'est pourquoi il faut se réjouir qu'on ait pris l'initiative de le réimprimer intégralement dans sa version originale. Cette édition de 1970 comporte également une nouvelle préface de Dunlop dans laquelle, en plus de citer de nombreux travaux réalisés à partir de son modèle théorique, l'auteur trace de nouvelles avenues de recherche afin de combler ce qui lui paraît être des lacunes dans l'application du modèle. En effet, dans les études comparatives au niveau international, les chercheurs ont surtout privilégié les variables politiques et idéologiques accordant moins d'importance dans leur analyse aux contextes de la technologie, d'une part, et du marché, d'autre part.

Dans quelle mesure, assistons-nous, dans un processus à long terme à une convergence des système nationaux de relations industrielles? Dans quelles circonstances, et dans quelle mesure, les institutions et les pratiques qui caractérisent les relations industrielles peuventelles être transplantées d'un pays à un autre ?

C'est sur ces grandes questions que l'auteur invite le lecteur à la poursuite de la recherche et de la réflexion théorique à l'heure des entreprises multinationales, des interdépendances économiques au plan international, de l'industrialisation lente mais certaine des pays en voie de développement.

Si l'ouvrage de Dunlop demeure un classique, tout n'est pas encore résolu dans l'élaboration d'une théorie des relations industrielles qui pourra rendre compte de cette réalité dans les conditions nouvelles où se pratiquent maintenant les relations industrielles.

Université Laval

Jean BERNIER

Nationalism and the Multinational Enterprise, by R. H. Hahlo, G. Smith et R. Wright, New York, Oceana Publications Inc., 1973, 373 pp.

«Nous sommes à l'âge de l'entreprise plurinationale » proclament les auteurs du livre dans leur préface. Il serait tout aussi exact de dire que nous sommes à l'âge des conférences sur l'entreprise plurinationale. Il n'est guère d'école supérieure, d'université, de gouvernement qui n'ait organisé sa conférence. Même les Nations Unies, par le truchement de leur Conseil Economique et Social, réunissaient récemment à New York des représentants de gouvernements affectés, d'entreprises plurinationales ainsi que des experts universitaires pour étudier ce problème. Il était donc logique que Montréal ait aussi «sa» conférence. Celle-ci a eu lieu à l'Université McGill, en août 1971. Ce sont les exposés présentés à cette occasion. étoffés par quelques études commanditées ultérieurement, qui composent l'ouvrage que nous nous efforcerons d'analyser.

Pour essayer de différencier cette conférence de la multitude des conférences analogues, les organisateurs ont eu l'idée de l'articuler autour du thème des relations entre l'entreprise plurinationale (E.P.N.) et le pays hôte et de développer les aspects légaux, politiques, économiques et administratifis de ces relations. L'idée était bonne et il est certain que l'entreprise plurinationale représente le sujet idéal pour une telle étude pluridisciplinaire. Malheureusement, il est très difficile, en pratique, d'équilibrer les contributions de chacune des disciplines et de les intégrer dans un tout homogène. Il suffit de jeter un coup d'oeil à la table des matières pour se rendre compte que l'aspect légal l'a nettement emporté sur les autres. En fait la grande majorité des rapports ont adopté une approche légale ou politique. L'aspect économique est très réduit, l'aspect management rudimentaire.

L'ouvrage se divise en cinq parties. La première partie étudie les réactions politico-légales des principaux pays ou groupements de pays à l'entreprise plurinationale. Ainsi, le professeur VAGTS, après avoir défini ce qu'il entend par entreprise plurinationale, indique la position des Etats-Unis face aux EPN. Il insiste tout particulièrement sur les domaines dans lesquels la législation américaine a des incidences extraterritoriales, domaines qui constituent des zones de conflit possibles avec les pays hôtes des filiales d'EPN américaines. Il analyse en détail l'incidence des lois interdisant les relations avec les pays du bloc communiste, ainsi que les questions de taxation, de lois antitrust, etc... Il 
montre également que si les Américains sont tout à fait partisans de la liberté d'action des EPN dans les autres pays, ils se gardent bien de mettre ces principes en application chez eux dans leurs relations avec les EPN étrangères.

La position du Canada est suffisamment bien connue pour que nous n'insistions pas sur ce point. Dans un très long exposé, M. Paul LELEUX parle des lois française et belge touchant à la constitution et au fonctionnement des EPN sur leur territoire.

R. Walter CHUDSON, dans son article intitule «Africa and the Multinational Enterprise », évoque la position générale des pays en voie de développement plutôt que celle de l'Afrique en particulier. Il indique, cependant, qu'en Afrique les EPN ont tendance à se concentrer dans les secteurs primaire et tertiaire, au détriment de l'industrie secondaire. Il souligne les points de conflits possibles avec les pays hôtes, mais termine sur une note optimiste : selon lui, il peut $y$ avoir bonne entente et profit mutuel, à condition que les pays hôtes exercent un contrôle vigilant sur certains aspects du fonctionnement des EPN. Cela ne signifie pas que les gouvernements doivent établir des contrôles minutieux et bureaucratiques sur l'activité des EPN, mais plutôt qu'ils doivent, par négociation, obtenir de ces dernières certains avantages comme la formation rapide de cadres, la transformation sur place des matières premières, etc. .

La deuxième partie se veut une évaluation des coûts et bénéfices économiques de l'EPN. En fait, elle tourne toute entière autour du conflit maintenant classique entre la position libérale traditionnelle soutenue par H.G. JOHNSON, dune part, la position nationaliste et restrictive d'Eric KIERANS, et de A. ROTSTEIN, de l'autre. L'incompatibilité des deux tendances est aussi entière après la conférence qu'avant, les adversaires ne parlant pas de la même chose. JOHNSON affirme que réduire le problème de l'opposition entre le nationalisme et l'EPN à un conflit entre bénéfices d'ordre économique et coûts d'ordre politique, c'est le simplifier outre mesure ; il reste, cependant, qu'on en revient toujours à cette opposition. JOHNSON adopte une position théorique : de même que dans le domaine du commerce international, il se prononce nettement pour la liberté totale de mouvement des marchandises, de même dans celui des investissements, il est partisan du laissez-faire absolu. Ce n'est que par cette liberté absolue, par la division du travail qui en résultera, que tous les pays retireront le maximum de profit de l'apport des EPN. Pour lui, l'EPN est un phénomène dynamique et positif, alors que les nations-états dans lesquelles elle s'implante sont des reliques du passé, totalement inadaptées aux conditions actuelles et imposant des discriminations inacceptables.

KIERANS et ROTSTEIN insistent au contraire sur les aspects politiques du débat. Les EPN ne sont responsables envers aucune autorité et ne cherchent que leur profit propre, au détriment du bien-être des sociétés dans lesquelles elles fonctionnent. On a surestimé leur apport économique : ainsi le Canada a le taux de chômage le plus élevé de tous les pays industrialisés bien qu'il ait sur son territoire le plus grand nombre d'EPN. D'autre part, la croissance économique n'est pas une panacée : elle apporte aussi des inconvénients et elle n'est qu'un des nombreux objectifs que toute société développée peut se fixer. Or, en acceptant trop d'EPN chez lui. un pays peut finir par perdre le contrôle sur les principales décisions affectant son économie, sa culture, ses relations avec les autres pays. En d'autres termes, les inconvénients de l'EPN sont de loin supérieurs aux avantages qu'elle apporte.

La troisième partie, sur l'administration (le 《management 》) des entreprises plurinationales n'est en fait que la continuation de la seconde partie dont elle reprend les thèmes principaux, peutêtre sur un plan plus pratique. Les trois articles qui la composent insistent encore plus que les précédents sur le conflit entre avantages et inconvénients de l'EPN, sur l'opposition irréductible entre partisans et adversaires de cette forme d'organisation. LITVAK et MAULE (Foreign Subsidiaires as an instrument of hostgovernment policy) et dans une moindre mesure, R. ROBINSON (Nationalism and centralized control) prennent le parti de l'état-nation, comme ROTSTEIN l'avait fait auparavant, et tentent de revaloriser le nationalisme, expression du caractère unique d'un peuple qui refuse de perdre son identité, 
même en échange d'avantages économiques, d'ailleurs mitigés. Ils soutiennent le droit fondamental de chaque peuple à fixer lui-même ses priorités sans interférences de l'extérieur et donc de contrôler l'activité des EPN qui voudraient lui imposer des décisions qui ne sont pas toujours dans son intérêt. Mais alors que LITVAK et MAULE suggèrent un contrôle assez pousíé (obligation de prendre des résidents locaux sur les conseils d'administration, obligation d'avoir des programmes de recherche locaux, publication des états financiers, etc...). ROBINSON craint que des mesures aussi sévères ne fassent fuir le capital étranger et suggère donc d'inciter les EPN à tenir compte des priorités locales au moyen d'incitations fiscales, douanières, etc. L'article de John FAYER WEATHER est l'antithèse de ces deux exposés; il reprend directement les arguments de H. G. JOHNSON, en les amplifiant. Pour lui, le pays hôte ne peut obtenir le maximum de profit de la présence des EPN qu'en les laissant totalement libres d'accomplir leur fonction économique. Tout subside, en introduisant des distortions dans le mécanisme du marché, diminue l'efficacité de l'EPN et donc le profit que le pays hôte en retire. Tout contrôle direct de ces entreprises les empêche d'accomplir leur mission et va donc à l'encontre de l'intérêt du pays hôte. Et il conclut : «... a significant degree of control of host national affairs by multinational firms is in fact in the interests of the hosts ;... much of the present attitudinal resistance is not sound ». On ne peut pas être plus net, ni aller plus loin dans le laisser faire.

Il ressort de cette confrontation entre partisans et adversaires de l'EPN que leurs théories sont absolument inconciliables. Pour ses partisans, l'EPN représente l'aboutissement ultime de la longue évolution des entreprises. L'entreprise plurinationale transcende les intérêts d'un seul pays pour s'intéresser au bienêtre mondial; par son activité, elle peut maximiser le bien-être économique du monde. Au contraire, l'état-nation est une relique du passé, un facteur de morcellement et de ségrégation entre les peuples. A l'opposé, les adversaires de l'EPN voient en elle une organisation qui n'a de compte à rendre à personne, qui profite de sa puissance pour jouer certains pays contre d'autres et pour les exploiter tous. Pour eux, le nationalisme, au contraire, est l'expression de la volonté des peuples, de leur refus à abdiquer leur identité.

Les quatrième et cinquième parties étudient principalement des problèmes légaux : les conflits, l'arbitrage et les relations industrielles dans la quatrième partie, les lois anti-trust dans la cinquième.

En définitive, ce volume n'apporte rien de vraiment nouveau. On n'y trouvera pas d'analyse théorique avancée ni de proposition pratique révolutionnaire. Il n'est cependant pas dénué de valeur, loin de là. En fait, il présente un double intérêt: d'abord il met en valeur la complexité du phénomène de l'entreprise plurinationale et la gamme incroyable des problèmes qu'elle soulève: problèmes légaux, politiques, sociaux, économiques, qui dépendent tous les uns des autres; ensuite, il permet de faire le point sur la question par un choix judicieux d'articles, certains soutenant et d'autres attaquant ces organisations.

\section{Université de Sherbrooke}

Gérard GARNIER

The Economics of Work and Pay, by Albert Rees, New York, Harper and Row, 1973, pp. ix, 247.

The subject matter of labor economics has been undergoing rapid and major changes on both the theoretical and empirical front in the last decade. Such changes have been all the more dramatic in an area which has traditionally avoided the analytical framework that characterizes the main body of economics. In this book, Albert Rees undertakes to outline and evaluate the techniques and results of the "new labor economics $\gg$ in a concise format designed for use by advanced undergraduates. The result is a timely and excellent book that I would judge an unqualified success in terms of both concept and clarity.

The book is organized into six parts that contain a total of sixteen chapters covering most of the problems that labor economists currently worry about. In part I, Rees discusses the factors affecting the quantity and quality of labor supply. This involves a detailed treat- 